\title{
EDITORIAL
}

\section{Bringing about lifestyle change}

O verwhelming evidence has firmly established the importance of lifestyle in the development of cardiovascular disease (CVD). Behavioral modifications, such as changing to a healthier diet, limiting alcohol consumption, increasing the amount and intensity of physical activity, or stopping smoking can have a huge impact in the primary prevention of CVD. In addition, adoption of these behaviors was shown to be as important as prophylactic pharmacotherapy in reducing the risk of major adverse cardiovascular events after acute coronary syndromes (Chow, C. K. et al. Circulation 12, 750-758; 2010). Awareness of the importance that lifestyle has on cardiovascular health has led to a wealth of public policy measures aimed at reducing lifestyle-related CVD risk. Organizations such as the UK's National Institute for Health and Clinical Excellence (http://www.nice.org.uk/ nicemedia/live/13024/49273/49273.pdf), the AHA (Lloyd-Jones, D. et al. Circulation 121, e46-e215; 2009), and the WHO (http://www.who.int/cardiovascular_ diseases/guidelines/Full\%20text.pdf) have advocated the promotion of behavioral change at the population level as one of the most effective measures to reduce the burden of CVD.

Unfortunately, a substantial proportion of people with behavioral risk factors for CVD are still not following their doctor's advice to quit smoking, start exercising, and eat more fruit and vegetables. Often, these people will not change their lifestyle until they suffer a stroke or myocardial infarction, and some will not even change after such events occur. This state of affairs is a source of great frustration for many health-care professionals, whose efforts are not being translated into improved patient health.

Many questions are being asked in the cardiology community about how patients' adherence to lifestyle modification programs can be improved. What part should be played by health-care professionals and the health-care system in trying to achieve these goals? Which aspects of their own health management should be left in the hands of the patient? After all, the ultimate goal is to make patients more responsible and careful about their well-being. Should clinicians focus on patient coaching? Should health-care organizations encourage patients by means of rewards (including monetary)? Alternatively, should certain forms of care be withheld if patients do not change their risky behaviors?

This latter question raises some ethical issues. If such a measure were adopted, would it not contradict a doctor's duty to treat patients as long as treatment is available? Who would decide when treatment should be denied? Which objective criteria would be used to weight such a decision? Most importantly, would such an incentive lead to the desired goal of making patients more responsible and, therefore, more careful with their own health? A good patient-provider relationship is known to be a major factor in increasing adherence to medication or behavioral change. The decision to withdraw care, at least when isolated from other measures, could potentially endanger this relationship and alienate patients from the health-care system. Furthermore, as ethnic minority and economically disadvantaged population groups have been claimed to have lower adherence rates and have also been found to be at increased risk of CVD (Stringhini et al. JAMA 303, 1159-1166; 2010), such an approach might disproportionately affect these patient groups.

On the other hand, the strategies that are currently in place to tackle the issue of behavioral change seem to be an insufficient answer to the magnitude of the problem, and the implementation of more-effective measures is urgently needed. The involvement of patients in the management of their health is certainly key to the success of any attempt to change behaviors. Awareness of the importance of this factor is reflected by the WHO recommendation of using "adherence" rather than "compliance" when referring to patient's attitudes towards medical or behavioral interventions.

A new statement from the AHA clearly demonstrates, however, that isolated approaches are unlikely to be very effective in changing behaviors linked to CVD risk (Artinian, N. T. et al. Circulation 122, 406-441; 2010). After assessing 74 studies published between 1997 and 2007 in the USA, the AHA researchers have concluded that a combination of approaches at the patient, provider, and health-policy levels yields better results than any isolated strategy. This statement could prompt the instigation of a coordinated strategy of CVD prevention that includes increased patient responsibility, improved resources for health-care providers to help patients change and maintain positive behaviors, better healthcare system policies to support patients and providers, and social fostering of behavioral change by legislators and private companies (for example, by implementing specific food regulations, promoting easy and cheap access to sports facilities, and/or lowering insurance premiums for those who adopt a healthier lifestyle). Perhaps governments and companies can be convinced to take part by emphasizing the potential financial gains associated with improved health. Hopefully, such a coordinated strategy will make difficult behavioral decisions easier to initiate and maintain.

doi:10.1038/nrcardio.2010.142

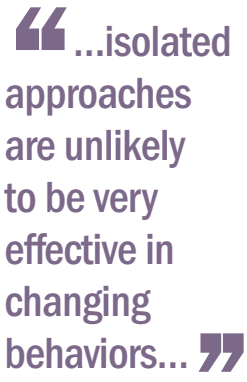

Joana Osório is the Associate Editor of Nature Reviews Cardiology.

Competing interests The author declares no competing interests. 